\title{
FLURALANER COMO TRATAMENTO PREVENTIVO DA DEMODICOSE GENERALIZADA EM CADELA EM PROESTRO: RELATO DE CASO
}

Marcos Antônio Celestino de Sousa Filho ${ }^{1}$; Hiran Esmeraldo Albuquerque Beserra ${ }^{2}$; Luanna Soares de Melo Evangelista ${ }^{3}$

${ }^{1}$ Aluno de Pós-graduação em Ciência Animal, Centro de Ciências Agrárias, Universidade Federal do Piauí (marcoscelestino90@gmail.com)

${ }^{2}$ Médico Veterinário Autônomo

${ }^{3}$ Prof ${ }^{a}$ Dr ${ }^{\text {a }}$ Departamento de Parasitologia e Microbiologia, Centro de Ciências da

Saúde, Universidade Federal do Piauí

Recebido em: 08/04/2017 - Aprovado em: 10/06/2017 - Publicado em: 20/06/2017

DOI: 10.18677/EnciBio_2017A54

\begin{abstract}
Este trabalho relata um caso de uma cadela domiciliada, da raça yorkshire, com um ano e cinco meses de idade, diagnosticada com demodicose. Ao entrar em proestro, previamente ao primeiro cio, a cadela apresentou lesões de pele em várias regiões do corpo, sendo levada para avaliação clínica e raspado de pele, foi diagnosticada com demodicose generalizada e tratada com Fluralaner (Bravecto ${ }^{\mathrm{TM}}$ ), por via oral, dose única. Após alguns dias do tratamento os sinais clínicos foram diminuindo, aumentando o crescimento dos pêlos nas áreas de alopecia. Antes de entrar no proestro seguinte, a cadela foi tratada preventivamente com o mesmo fármaco, na mesma dosagem e frequência de administração, não apresentando nenhum sinal clínico da doença. Portanto o Bravecto ${ }^{\mathrm{TM}}$ pode ser administrado de forma preventiva para a demodicose em cadelas que estão em proestro, caracterizando um possível uso do animal à reprodução.
\end{abstract}

RESUMO

PALAVRAS-CHAVE: acaricida, alteração hormonal, canino, demodicose.

\section{FLURALANER AS A PREVENTIVE TREATMENT FOR GENERALIZED DEMODICOSIS IN BITCH IN PROESTRO: CASE REPORT}

\begin{abstract}
This paper reports a case of a domiciled bitch, Yorkshire breed, one year and five months old, diagnosed with demodicosis. When entering proestrus, before first estrus, the bitch showed skin lesions in many regions of the body. After clinical evaluation and skin scraping, generalized demodectic mange was diagnosed and treated with Fluralaner (Bravecto ${ }^{\mathrm{TM}}$ ) single dose, orally. After a few days of treatment, there was a reduction of clinical signs and the beginning of hair growth in alopecia areas. Before entering the next proestrus, the dog was treated preventively with the same drug at the same dosage and frequency of administration presenting no clinical signs of the disease. Therefore Bravecto ${ }^{\mathrm{TM}}$ can be administered in a preventive way for demodicosis in dogs that are in proestrus, characterizing a possible use of the animal for reproduction.
\end{abstract}

KEYWORDS: acaricide, hormonal alteration, canine, demodicosis. 


\section{INTRODUÇÃO}

A demodicose canina ou sarna demodécica é uma dermatopatia parasitária inflamatória causada pela proliferação do Demodex spp., ácaro comensal da pele de cães, que parasita dentro do folículo piloso e glândulas sebáceas, se alimentando de células epiteliais do hospedeiro. É um parasita que só prolifera causando lesões quando ocorre redução da imunidade (MONTEIRO, 2014; FOURIE et al., 2015).

Segundo FUKAHORI et al. (2013) as dermatopatias têm considerável presença nos consultórios veterinários, sendo a demodicose uma das mais frequentes. A doença pode ser classificada como localizada ou generalizada, de acordo com a extensão das lesões e pode ocorrer na fase juvenil (até 18 meses) ou vida adulta (FUKAHORI et al., 2013; FOURIE et al., 2015).

A forma localizada ocorre em pequenas áreas de alopecia, eritematosas, circunscritas, escamosas, podendo ser pruriginosas ou não, mais comumente observadas na face e nas patas dianteiras, sendo de resolução espontânea. A demodicose generalizada se apresenta como uma dermatite crônica com liquenificação, descamação, formação de crostas, hiperpigmentação, piodermatite severa e alopecia, cobrindo grandes áreas do corpo (DELAYTE et al., 2006).

Inúmeros fatores influenciam a suscetibilidade dos cães às infestações por Demodex spp: doenças que afetam o sistema imunológico provocando a supressão do mesmo, endoparasitismo, diferentes raças, idade, situação hormonal ou nutricional e outras doenças imunológicas derivadas de defeitos genéticos e alteração da bioquímica e da estrutura da pele (SINGH \& DIMRI, 2014).

O proestro nas cadelas surge com uma alteração hormonal do ciclo estral em que há uma elevada concentração de estrógeno, promovendo alteração em diversos órgãos reprodutivos (FELDMAN \& NELSON, 2004). Durante essa fase, ocorrem também mudanças comportamentais na fêmea, ela se torna mais ativa e esse estresse transitório pode exacerbar ou recidivar a demodicose generalizada (LEITÃO \& LEITÃO, 2008).

O uso de amitraz tópico era o tratamento mais indicado para a demodicose, porém, nem sempre era efetivo e bem tolerado. Outras alternativas terapêuticas as situações de insucesso no tratamento com esse fármaco foram surgindo e novas drogas passaram a ser mais utilizadas, tais como: ivermectina, milbemicina oxima, moxidectina e lufenuron, normalmente requerendo uma grande adesão do tutor a um período de tratamento prolongado (PARADIS, 1999; PATERSON et al., 2014).

O fluralaner, princípio ativo do Bravecto ${ }^{T M}$, é um novo inseticida e acaricida sistêmico, de longa duração, que pertence a família da isoxazolina, classe dos parasiticidas com inibição seletiva dos canais do ácido $\mathrm{Y}$-aminobutírico e do Lglutamato cloroligante dos artrópodes (GASSEL et al., 2014). Em estudos de campo envolvendo cães com demodicose generalizada, uma única administração oral de Bravecto $^{\text {TM }}$, mostrou ser $100 \%$ eficaz contra a doença e sem ácaros detectáveis aos 56 e 84 dias após o tratamento (FOURIE et al., 2015).

Desta forma, este trabalho objetivou descrever um relato de caso de uma cadela diagnosticada com demodicose generalizada, que foi tratada preventivamente com Fluralaner na fase de proestro.

\section{DESCRIÇÃO DO CASO}

Este trabalho descreve uma cadela domiciliada, da raça yorkshire, de um ano e cinco meses de idade. Ela foi diagnosticada pela primeira vez com demodicose 
generalizada no início de junho de 2016, com quase nove meses de idade, durante a fase de proestro, previamente ao primeiro cio.

A cadela foi levada ao atendimento veterinário, e foram realizados exame clínico e raspado cutâneo profundo, por ser esta a melhor opção para determinar a presença do ácaro, além de fácil execução, baixo custo e alta sensibilidade. $O$ material coletado foi posteriormente depositado em lâmina com óleo mineral e observado em microscópio óptico (40x) confirmando a presença de Demodex canis.

Realizou-se o tratamento com a administração de Bravecto $^{T M}$, na dosagem única e em comprimido para cães de 2 a 4,5 quilos, por via oral, sendo observada redução das lesões de pele, redução de prurido e ressecamento das regiões acometidas 10 dias após a administração do medicamento.

Um mês após o tratamento observou-se uma melhora considerável no quadro clínico da doença com o fim dos sinais clínicos previamente apresentados e o crescimento de pêlos nos locais acometidos. Em dezembro de 2016, ainda em anestro, a cadela foi tratada preventivamente contra demodicose com o mesmo fármaco, na mesma dosagem e frequência de administração, com o intuito de não apresentar os sinais clínicos da doença no proestro seguinte.

\section{RESULTADOS E DISCUSSÃO}

Segundo FUKAHORI et al. (2013) as dermatopatias têm considerável presença nos consultórios veterinários, sendo a demodicose uma das mais frequentes. De acordo com SOCOLOSKI et al. (2015) esta doença é uma enfermidade causada pela proliferação exacerbada do ácaro Demodex canis, sendo classificada como adulta ou juvenil e localizada ou generalizada.

O animal deste caso clínico apresentava alguns fatores predisponentes para a ocorrência da demodicose, como a raça e a idade. Segundo RIBEIRO (2013) e KHOSHNEGAH et al. (2013) o yorkshire é uma raça que tem maior frequência na consulta dermatológica e cães Terrier apresentam um risco aumentado de doença dermatológica.

Previamente ao diagnóstico da demodicose, a cadela apresentou lesões de pele em várias regiões do corpo, sendo mais intensas na face, membros anteriores e dorso. As lesões foram se intensificando e generalizando o processo em menos de um mês, condizendo com caso crônico e corroborando com outros relatos descritos na literatura (FUKAHORI et al., 2013).

As lesões encontradas no animal apresentaram-se como áreas de alopecia com descamações e crostas no focinho, face periocular e em ambos os membros anteriores, além de apresentar foliculite e piodermatite na região dorsal como mostra a Figura 1, corroborando com GAMITO (2009), que descreve a demodicose com presença de lesões redondas alopécicas, com vários graus de eritema, ocorrendo também descamação, com distribuição lesional nas regiões peri-ocular, peri-bucal e espaços interdigitais dos membros anteriores. 

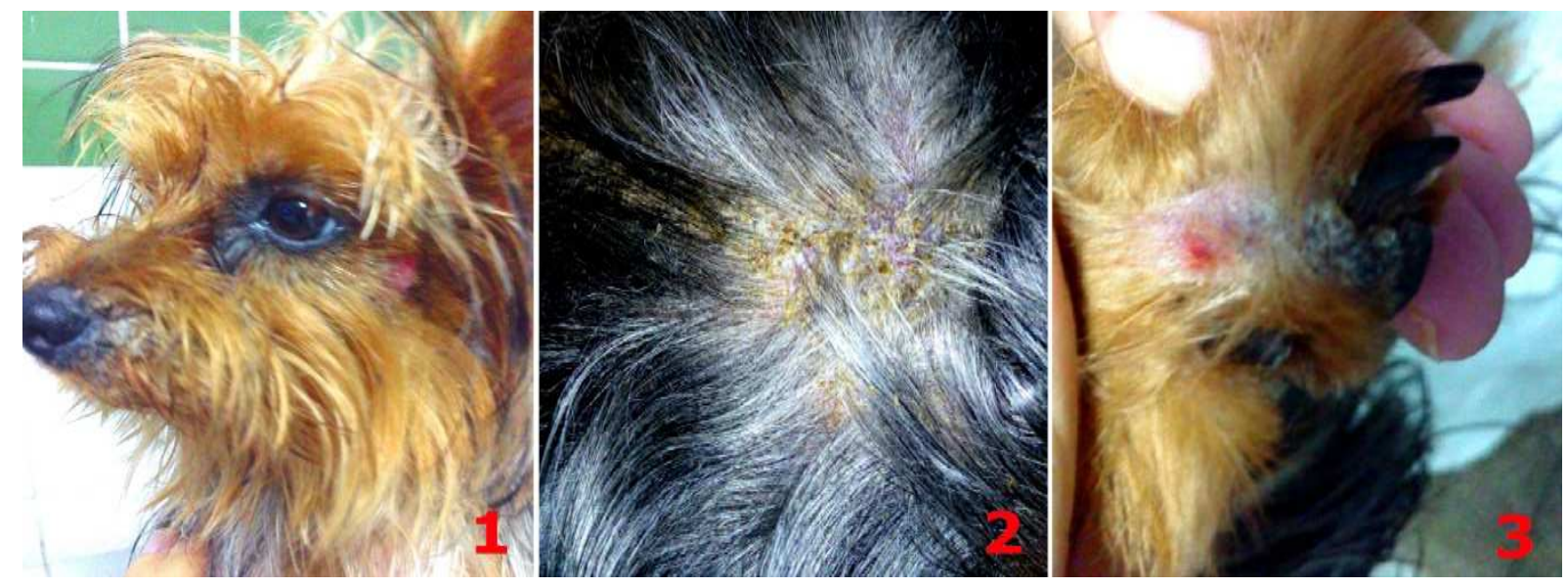

FIGURA 1. Características das lesões de Demodicose Generalizada. 1 - Lesão facial na região periocular e focinho; 2 - Lesão na região dorsal; 3 - Lesão na região do membro dorsal esquerdo.

Fonte: autores

O diagnóstico foi realizado quando a cadela entrou em proestro, antes do primeiro cio, condição que a literatura relata ser de intensa atividade hormonal e, portanto, pode causar baixa imunidade em fêmeas caninas (LEITÃO \& LEITÃO, 2008). A Figura 2 mostra a presença do ácaro no raspado cutâneo profundo realizado no animal relatado.

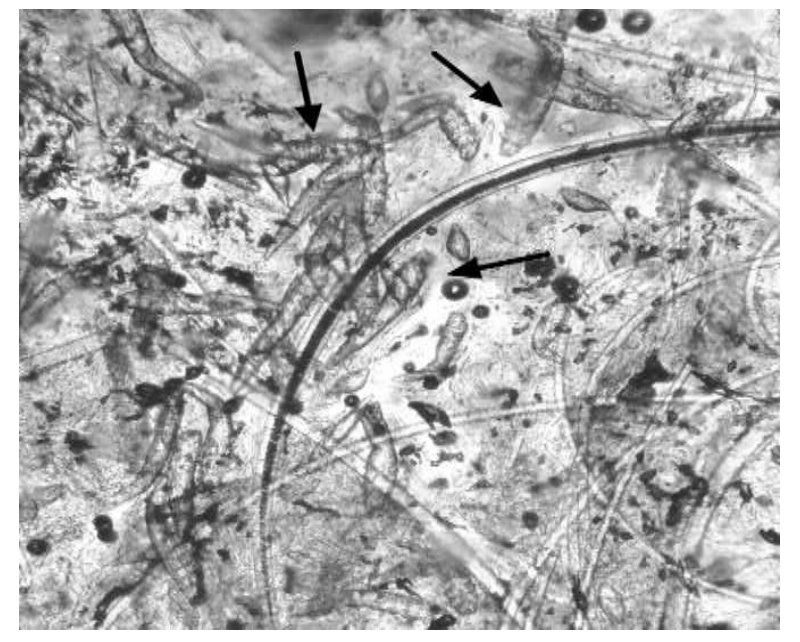

FIGURA 2. Lâmina do raspado cutâneo profundo em microscopia óptica com o aumento de 40x, mostrando a presença de Demodex spp. no animal.

Fonte: autores

Após o diagnóstico da demodicose, a cadela foi tratada com o Bravecto ${ }^{\mathrm{TM}}$ para redução das lesões e a observação da melhora clínica se deu alguns dias após o tratamento, como mostra a Figura 3. 

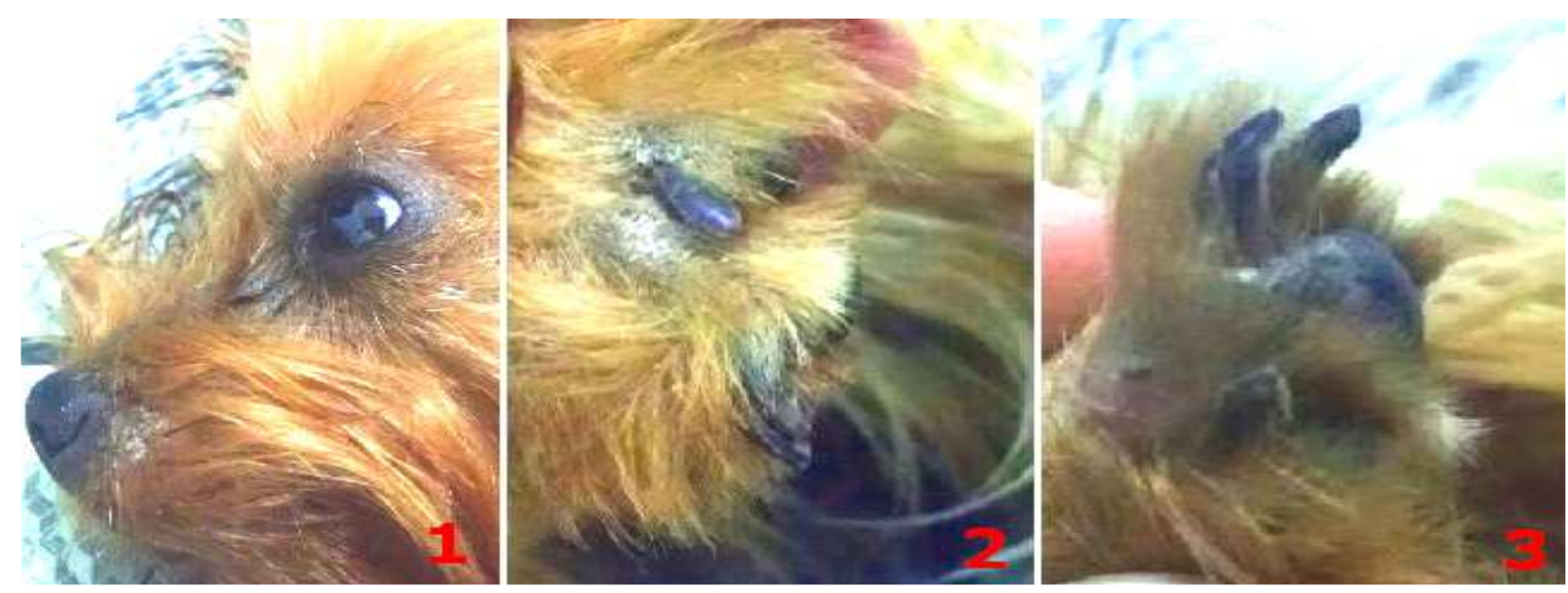

FIGURA 3. Características das lesões dez dias após o tratamento com Bravecto ${ }^{\mathrm{TM}}$. 1 - Lesão facial na região periocular e focinho; 2 - Lesão na região do membro dorsal direito; 3 - Lesão na região do membro dorsal esquerdo.

Fonte: autores

Com o passar dos dias foi observada a resolução das crostas e das descamações e crescimento de novos pêlos nas regiões alopécicas, também não foram mais observados ácaros ao raspado de pele, confirmando os achados da lieratura (FOURIE et al., 2015). A fim de diminuir a possibilidade de recidiva da doença, foi administrado o mesmo fármaco seis meses após, com a cadela ainda em anestro. Em janeiro de 2017 ela entrou em proestro e posteriormente no estro, e não foi observada nenhuma manifestação clínica da demodicose, conforme se observa na Figura 4.

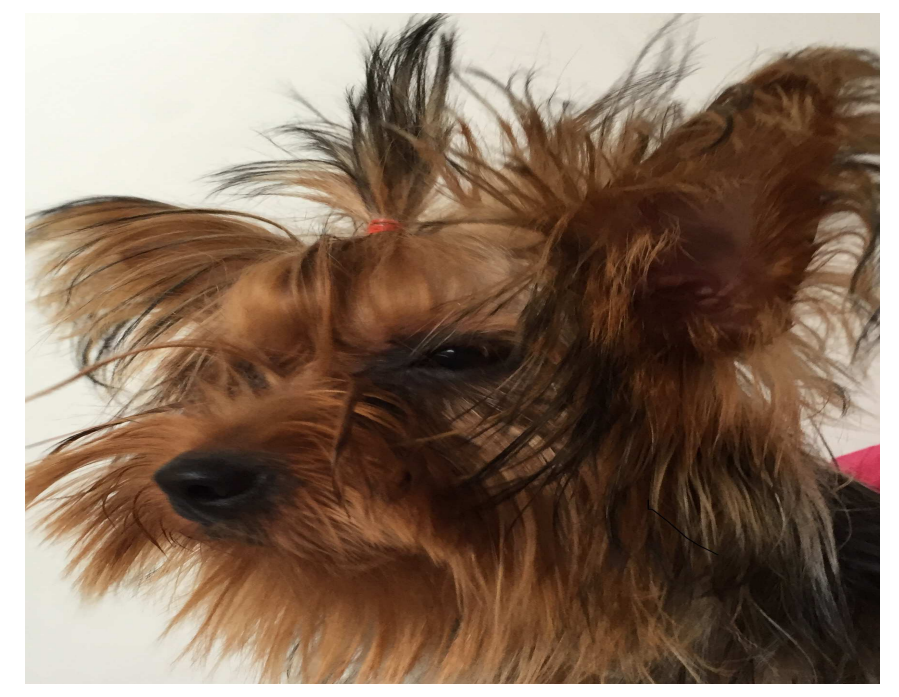

FIGURA 4. Animal sem lesão característica de Demodicose.

Fonte: autores

FUKAHORI et al. (2013) mostraram que os raspados de pele realizados em cães após oito semanas de tratamento com moxidectina, via oral, foram negativos para Demodex canis, enquanto que DELAYTE et al. (2006) indicaram que o primeiro 
exame negativo do exame de pele em cadela tratada para a demodicose se deu aos 150-154 dias de tratamento utilizando a moxidectina na dose $0,5 \mathrm{mg} / \mathrm{kg}$, injetável.

O uso de Bravecto ${ }^{\mathrm{TM}}$, por via oral, como forma preventiva da demodicose em cadelas que estão entrando em estro é de grande relevância para que as mesmas não sejam totalmente afastadas da reprodução, já que o caráter hereditário da doença não está definitivamente comprovado.

\section{CONCLUSÃO}

Cadelas com demodicose tratadas preventivamente com Fluralaner (Bravecto ${ }^{\mathrm{TM}}$ ) em fase de proestro podem não apresentar sinais clínicos de lesões de pele durante essa fase hormonal, sendo importante a realização de mais estudos visando avaliar esse potencial de ação do fármaco em questão.

\section{REFERÊNCIAS}

DELAYTE E. H.; OTSUKA M.; LARSSON, C. E.; CASTRO, R. C. C. Eficácia das lactonas macrocíclicas sistêmicas (ivermectina e moxidectina) na terapia da demodicidose canina generalizada. Arquivo Brasileiro de Medicina Veterinária e Zootecnia, v. 58, n. 1, p. 31-38, 2006. Disponível em: http://www.scielo.br/pdf/abmvz/v58n1/28777.pdf

doi: $10.1590 / S 010209352006000100006$

FELDMAN, E. C.; NELSON, R. W. Canine and feline endocrinology and reproduction. $3^{\underline{a}}$ ed. Philadelphia: W. B. Saunders, 2004. 1089p.

FOURIE, J. J.; LIEBENBERG, J. E.; HORAK, I. G.; TAENZLER, J.; HECKEROTH, A. R.; FRÉNAIS, R. Efficacy of orally administered fluralaner (Bravecto ${ }^{\mathrm{TM}}$ ) or topically applied imidacloprid/moxidectin (Advocate®) against generalized demodicosis in dogs. Parasites \& Vectors, v. 8, p. 1-7, 2015. Disponível em: <https://www.ncbi.nlm.nih.gov/pmc/articles/PMC4394402/>

doi: 10.1186/s13071-015-0775-8

FUKAHORI, F. L. P.; LIMA, E. R.; REGO, M. S. A.; DIAS, M. B. M. C.; FERREIRA, M. A. Q. B.; SILVA, V. C. L.; LEITÃO, R. S. C. S. Eficácia do uso de moxidectina por via oral no tratamento de demodicose generalizada em cães: breve relato de dois casos. Medicina Veterinária, Recife, v. 7, n. 1, p. 31-34, 2013. Disponível em: <http://www.journals.ufrpe.br/index.php/medicinaveterinaria/article/viewFile/603/482>

GAMITO, M. S. R. Dermatites parasitárias no cão. 2009. 87p. Dissertação (Mestrado Integrado em Medicina Veterinária), Universidade Técnica de Lisboa Faculdade de Medicina Veterinária, Lisboa, 2009. Disponível em: <https://www.repository.utl.pt/bitstream/10400.5/1237/1/Dermatites\%20Parasit\%C3\% A1rias\%20no\%20C\%C3\%A3o.pdf>

GASSEL, M.; WOLF, C.; NOACK, S.; WILLIAMS, H.; ILG, T. The novel isoxazoline ectoparasiticide fluralaner: Selective inhibition of arthropod $\mathrm{y}$-aminobutyric acidand $\mathrm{L}$ glutamategated chloride channels and insecticidal/acaricidal activity. Insect Biochemistry and Molecular Biology, v. 45, p. 111-24, 2014. Disponível em:<https://www.ncbi.nlm.nih.gov/pubmed/24365472> doi: 10.1016/j.ibmb.2013.11.009. 
KHOSHNEGAH, J.; MOVASSAGHI, A. R.; RAD, M. Survey of dermatological conditions in a population of domestic dogs in Mashhad, northeast of Iran (20072011). Veterinary Research Forum, v.4, n.2, p. 99-103, 2013. Disponível em: <https://www.ncbi.nlm.nih.gov/pubmed/25653779>

LEITÃO, J. P. A.; LEITÃO, J. P. A. Demodicose canina. Revista Portuguesa de Ciências Veterinárias, v. 103, n. 567-568, p. 135-149, 2008. Disponível em: <http://www.fmv.ulisboa.pt/spcv/PDF/pdf12_2008/135-149.pdf>

MONTEIRO, S. G. Parasitologia na Medicina Veterinária. 1aㅡ ed. São Paulo: Roca, 2014. 356p.

PARADIS, M. New approaches to the treatment of canine demodicosis. Veterinary Clinics of North America: Small Animal Practice, v.29, p.1425-1436, 1999. Disponível em: <http://www.sciencedirect.com/science/article/pii/S0195561699501368> doi: 10.1016/S0195-5616(99)50136-8

PATERSON, T. E.; HALLIWELL, R. E.; FIELDS, P. J.; LOUW, M. L.; BALL, G.; LOUW, J.; PINCKNEY, R. Canine generalized demodicosis treated with varying doses of a $2.5 \%$ moxidectin $+10 \%$ imidacloprid spot-on and oral ivermectin: Parasiticidal effects and long-term treatment outcomes. Veterinary Parasitology, v. 205, n. 3-4, p. 687-96, 2014. Disponível em: <https://www.ncbi.nlm.nih.gov/pubmed/25262617> doi: 10.1016/j.vetpar.2014.08.021

RIBEIRO, C. P. C. C. Citologias e Biópsias Cutâneas: Estudo Estatístico Retrospetivo de 2009 a 2013. 2013. 81p. Dissertação (Mestrado Integrado em Medicina Veterinária). Universidade de Trás-os-Montes e Alto Douro. Vila Real, 2013. Disponível em: < https://repositorio.utad.pt/handle/10348/4637>

SINGH, K. S.; DIMRI, U. The immuno-pathological conversions of canine demodicosis. Veterinary Parasitology, v. 203, p. 1-5, 2014. Disponível em: <https://www.ncbi.nlm.nih.gov/pubmed/24680602>

doi: 10.1016/j.vetpar.2014.03.008

SOCOLOSKI, S. N. G.; BUREMA, M. C.; MONTEIRO, B. G.; VECCHIA, A. M. D.; ZORZO, C.; ALMEIDA, E. C. P.; BRAGA, A. P.; VIEIRA, T. B. Demodiciose generalizada adulta e juvenil: Relato de dois casos. Veterinária e Zootecnia, n. 22, v. 3, p. 386-391, 2015. Disponível em: < http://www.fmvz.unesp.br/rvz/index.php/rvz/article/view/960> 